\title{
Myxoid Solitary Fibrous Tumor on the Scalp
}

\author{
Ji Hyun Kim ${ }^{1}$, \\ Dong Chul Kim ${ }^{1}$, \\ Ryun Lee ${ }^{1}$, \\ Chi Ho Shin ${ }^{1}$, \\ Yea Sik Han', \\ Sang Hun Chung ${ }^{1}$, \\ So Ya Paik ${ }^{2}$ \\ Departments of ${ }^{1}$ Plastic and Reconstructive \\ Surgery and ${ }^{2}$ Pathology, Bundang Jesaeng \\ Hospital, Seongnam, Korea
}

\begin{abstract}
Myxoid solitary fibrous tumor (SFT) is very rare soft tissue neoplasm. It is microscopically composed of spindle cells which is individually separated by delicate band of collagen fibers. And this tumor cells are immunohisto-chemistrically highlighted by CD34. Myxoid SFT has indolent clinical course and a good prognosis, so it is important to make a diagnosis because of its morphological similarities to myxoid spindle cell neoplasms that have different prognoses and treatment. We report the case of a 20 -year-old female with a myxoid SFT found in the left temporo-parietal scalp. This case report appears to be the first reported scalp occurrence of this rare tumor.
\end{abstract}

Keywords: Neoplasm / Myxoid / Solitary fibrous tumors / Spindle cell / CD34

\section{INTRODUCTION}

Solitary fibrous tumor (SFT) is a rare benign spindle-cell neoplasm, which is composed of small cells with dense or vesicular nuclei and scant cytoplasm individually separated by thin bands of collagen fibers. SFTs are grossly circumscribed but frequently infiltrate microscopically [1]. And they are usually found in adults and occur in a wide range of anatomic sites [2]. Their size range from 0.8 to $26 \mathrm{~cm}$, and they may recur but rarely metastasize. The SFTs with malignant histologic features such as high cellularity, areas of necrosis, tumor size $(>10 \mathrm{~cm})$, high mitotic count $(>4 / 10$ high power field), and atypical mitotic figures are more likely to recur and metastasize [3,4]. Immunohistochemically, they are positive for CD34 and negative for smooth muscle actin, desmin, S-100 protein.

Focal myxoid change is a well-known characteristic of SFT. However, SFT with extensive myxoid stroma has been only reported occasionally. Myxoid SFT is first described by de Saint Aubain Somerhausen et al. [5] in 1999 as an SFT with myxoid change in $50 \%$ or more of the tumor. Recognition of this uncommon SFT

Correspondence: Dong Chul Kim

Department of Plastic and Reconstructive Surgery, Bundang Jesaeng Hospital, 20

Seohyeon-ro, 180 beon-gil, Bundang-gu, Seongnam 13590, Korea

E-mail: medicalinternet@yahoo.co.kr

Received Aug 9, 2017 / Revised Oct 27, 2017 / Accepted Nov 20, 2017 is important in order to distinguish a variety of myxoid spindle cell neoplasm with different biologic potential. This study reports a case of a young woman who had a rare myxoid solitary tumor on left temporo-parietal scalp area.

\section{CASE REPORT}

A 20-year-old female patient presented to our hospital with a fouryear history of a recently growing painless lump on left scalp (Fig. 1). There was no specific medical history and no tenderness on physical examination. The mass had a round shape and was palpated softly but not as soft as lipoma. Ultrasonography examination showed $4.1 \times 1.4 \mathrm{~cm}$ sized well-defined ovoid mass lesion with mild compressibility on subgaleal layer of left scalp area and no vascularity on Doppler. And well-defined soft tissue mass with no fat content was observed on the facial computed tomography (CT) image (Fig. 2). The lesion was completely resected under general anesthesia. This soft tissue mass was found on the subgaleal layer and firmly attached to the periosteum. The mass was grossly well circumscribed (Fig. 3). It was divided into halves, and it showed a bulging pale myxoid cut surface. Histopathological examination revealed bland oval to short spindle cell proliferation in a patternless pattern admixed with ropy collagen and numerous thinwalled vessels in a myxoid background. No necrosis was present. 


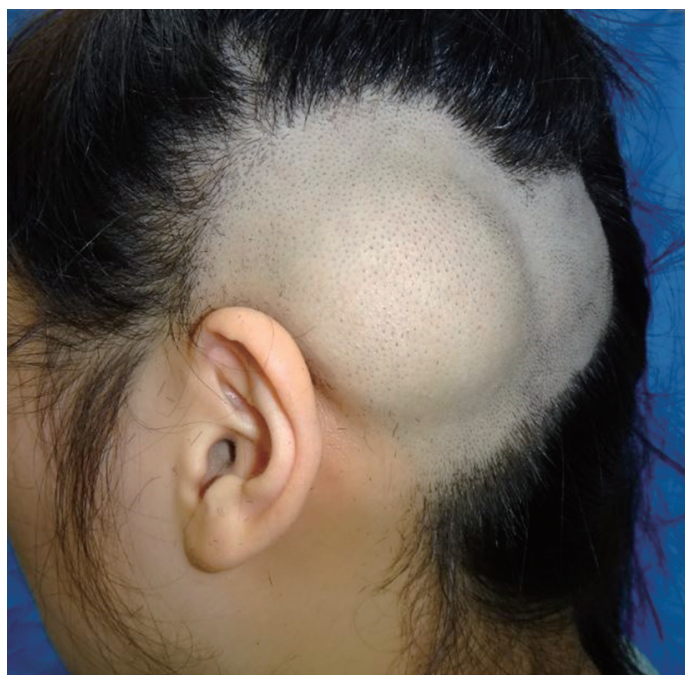

Fig. 1. Preoperative view. Preoperative photograph of a 20 -year-old female with about $5 \mathrm{~cm}$ round bulging mass on left temporo-parietal scalp.

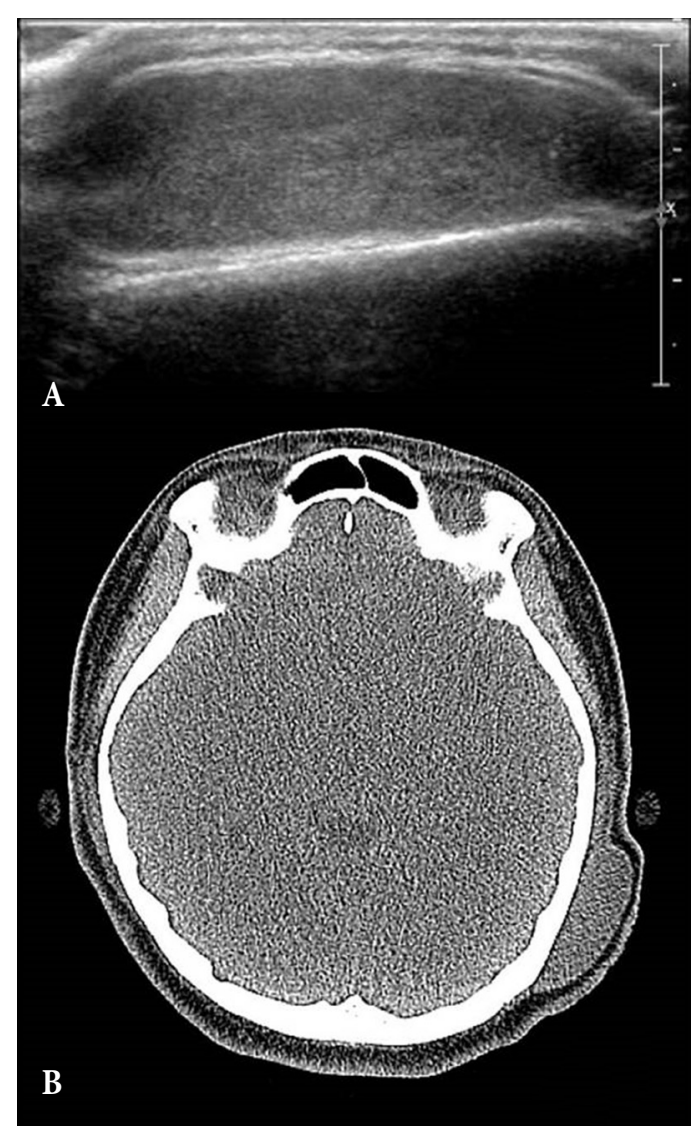

Fig. 2. Preoperative imaging. (A) Ultrasonography examination showed $4.1 \times 1.4 \mathrm{~cm}$ sized well-defined ovoid mass lesion. (B) Facial computed tomography image demonstrated the well-defined soft tissue mass with no fat content.
Immunohistochemically, it was positive for CD34 and negative for BCL2, smooth muscle actin, S-100 protein, and desmin (Fig. 4). The clinical manifestation and histological findings were most consistent with a diagnosis of myxoid SFT. There was no evidence of recurrence or metastasis during the 6-month follow-up period (Fig. 5).

\section{DISCUSSION}

SFT is a rare mesenchymal neoplasm that is postulated to arise from ubiquitous interstitial stem cells situated within soft tissues [6]. Tumours cells in SFT are characteristically immunoreactive for CD34. 20 to $35 \%$ of them are also variably positive for epithelial membrane antigen, BCL2, and smooth muscle actin [7]. Focal myxoid change in SFT is common, but predominantly myxoid SFT are extremely rare. Following the initial series of myxoid SFT described by de Saint Aubain Somerhausen et al. [5], only a few cases of myxoid SFT have been described. Tumor locations included pleura, orbit, and periparotid subcutaneous tissue, as well as four cases in deep soft tissue, two in the abdominal wall and one each in the chest wall and thigh. Dantey and Cooper [1] reported three cases of mxyoid SFT. Yap et al. [8] reported a case of myxoid SFT of the ischiorectal fossa. Chang et al. [9] reported a case of myxoid SFT of the upper thigh, and Lee et al. [10] reported

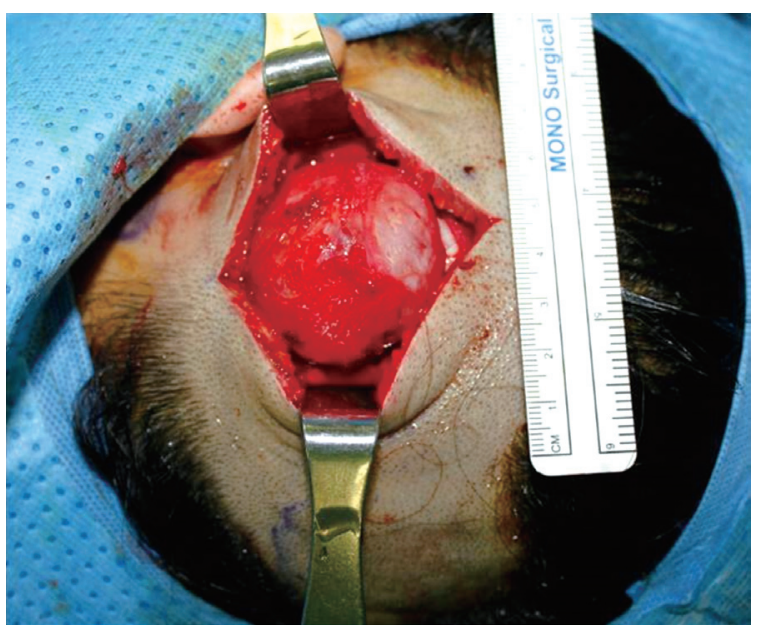

Fig. 3. Intraoperative view. Intraoperative. About $4 \times 4 \times 1.5 \mathrm{~cm}$ sized round mass under the subgaleal space was exposed. 

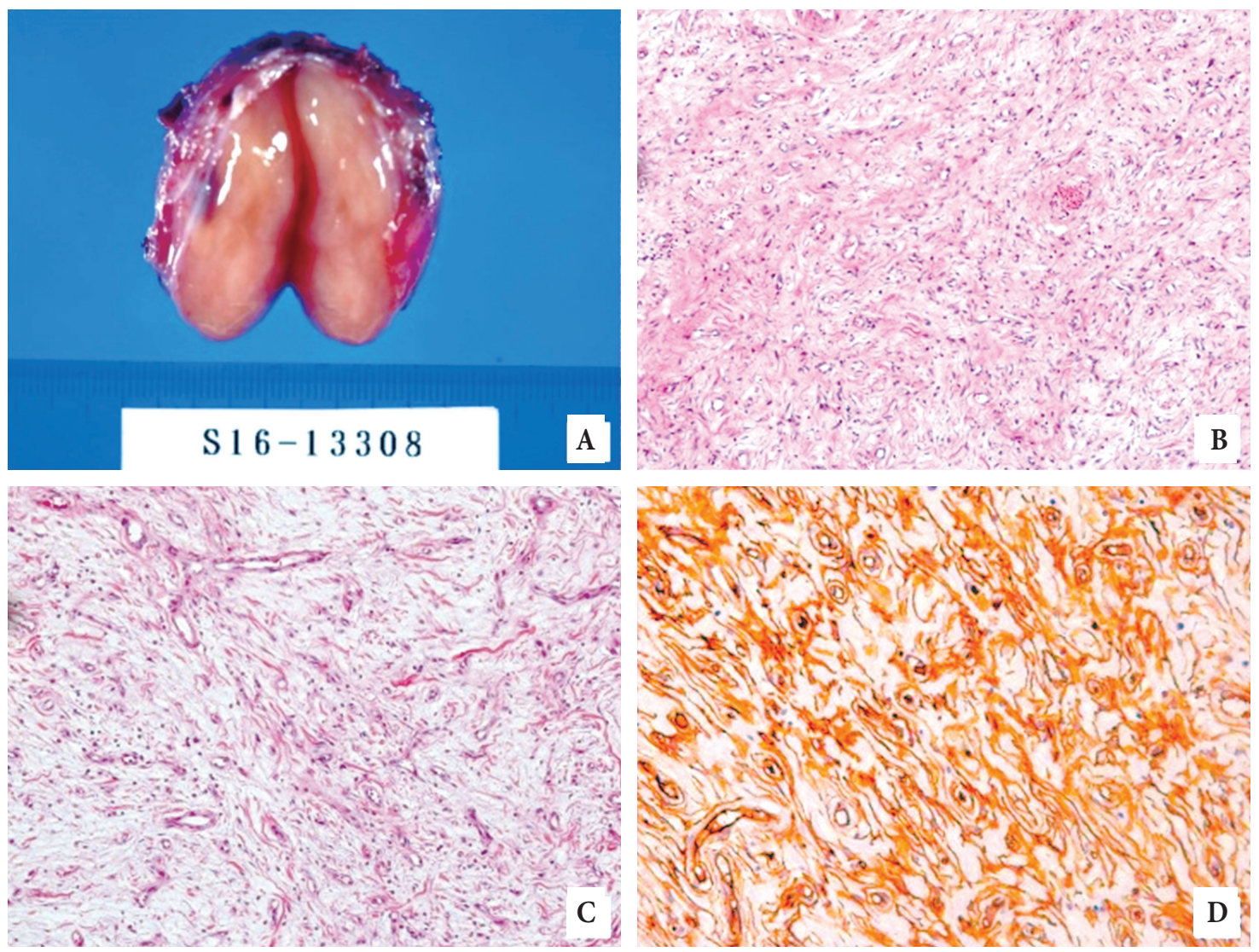

Fig. 4. Gross photo of the surgical specimen and histopathological finding. (A) Well circumscribed solid mass with a bulging pale myxoid cut surface without hemorrhage or necrosis. (B) Bland oval to short spindle cells dispersed in a patternless pattern among thin collagen bands and numerous small thin-walled vessels $(\mathrm{H} \& \mathrm{E}, \times 100)$. (C) Hypocellular myxoid areas showing thin ropy collagen bands $(\mathrm{H} \& \mathrm{E}, \times 200)$. (D) Immunohistochemical stain showing diffuse intense positivity to CD $34(\times 200)$.

a case of myxoid SFT of the left palm.

Although SFT was originally described as a pleural tumor, increasing number of extrapleural SFTs were reported. But SFTs of the head and neck region are particularly rare and most commonly benign. Myxoid SFT on scalp has never been reported before, so this case is a first reported scalp occurrence of this rare tumor.

The diagnosis of myxoid SFT can be challenging, because of its rarity and morphological features that overlap with those of a wide range of myxoid spindle cell neoplasm. Microscopically, a myxoid SFT lesion is characterized by abundant pale myxoid stroma, staghorn blood vessels and a patternless pattern arrangement of cytological bland spindle cells, that are singly separated by thin bland of collagen fibers [11]. The diagnosis depends on

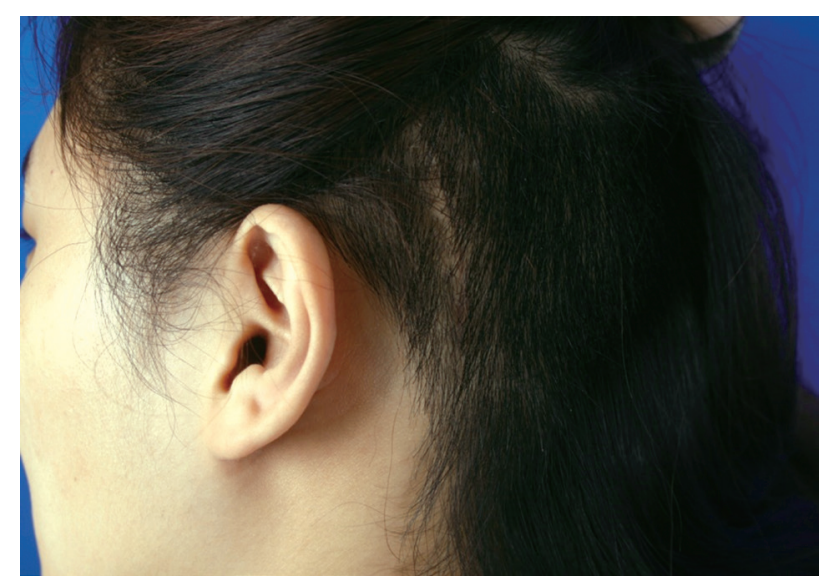

Fig. 5. Postoperative view. Postoperative 6 months follow-up. No recurrence was noted. 
microscopic and immunohisto-chemical features, although imaging may help. Its recognition is crucial because of its morphological similarities to myxoid spindle cell neoplasms that have different prognoses and treatment options. Patients with these tumors can be safely treated with local excision. According to the results of the studies, there is still no case of tumor recurrence or metastasis [9-12]. But tumors with positive margins require close follow-up over several years owing to the potential for late local recurrence.

\section{CONFLICT OF INTEREST}

No potential conflict of interest relevant to this article was reported.

\section{PATIENT CONSENT}

The patient provided written informed consent for the publication and the use of their images.

\section{REFERENCES}

1. Dantey K, Cooper K. Myxoid solitary fibrous tumor: a study of three cases. Int J Surg Pathol 2013;21:358-62.

2. Chan JK. Solitary fibrous tumour: everywhere, and a diagnosis in vogue. Histopathology 1997;31:568-76.

3. England DM, Hochholzer L, McCarthy MJ. Localized benign and malignant fibrous tumors of the pleura: a clinicopathologic review of 223 cases. Am J Surg Pathol 1989;13:640-58.

4. Gold JS, Antonescu CR, Hajdu C, Ferrone CR, Hussain M, Lewis JJ, et al. Clinicopathologic correlates of solitary fibrous tumors. Cancer 2002;94:1057-68.

5. de Saint Aubain Somerhausen N, Rubin BP, Fletcher CD. Myxoid solitary fibrous tumor: a study of seven cases with emphasis on differential diagnosis. Mod Pathol 1999;12:463-71.

6. Gengler C, Guillou L. Solitary fibrous tumour and haemangiopericytoma: evolution of a concept. Histopathology 2006;48:63-74.

7. Christopher DM, Krishnan U, Fredrik M. Pathology and genetics of tumours of soft tissue and bone. Lyon: IARCPress; 2002.

8. Yap T, Hamzah L, Oshowo A, Taylor I. Myxoid solitary fibrous tumour of the ischiorectal fossa. Eur J Surg Oncol 2003;29:98-100.

9. Chang SE, Bae GY, Choi JH, Sung KJ, Moon KC, Koh JK, et al. Cutaneous solitary fibrous tumour with myxoid stroma. Br J Dermatol 2002;147:1267-9.

10. Lee JY, Park SE, Shin SJ, Kim CW, Kim SS, Kim KH. Solitary fibrous tumor with myxoid stromal change. Am J Dermatopathol 2015;37: 570-3.

11. Vallat-Decouvelaere AV, Dry SM, Fletcher CD. Atypical and malignant solitary fibrous tumors in extrathoracic locations: evidence of their comparability to intra-thoracic tumors. Am J Surg Pathol 1998; 22:1501-11.

12. Ganly I, Patel SG, Stambuk HE, Coleman M, Ghossein R, Carlson D, et al. Solitary fibrous tumors of the head and neck: a clinicopathologic and radiologic review. Arch Otolaryngol Head Neck Surg 2006;132: 517-25. 